\title{
CERÁMICA GRABADA TARTÉSICA DEL CARAMBOLO: NUEVOS TESTIMONIOS*
}

\section{TARTESSIC CARAMBOLO RECORDED CERAMIC: NEW EVIDENCE}

\author{
MANUEL CASADO ARIZA
}

\begin{abstract}
Resumen: Con este trabajo pretendemos, simplemente, dar a conocer nuevos datos referentes a la cerámica con decoración grabada tartésica. Concretamente los testimonios documentados en el yacimiento del Carambolo durante las campañas 2002-2005. Concluiremos con una serie de reflexiones acerca de este tipo cerámico, sobre su funcionalidad y contenido simbólico, así como las implicaciones étnicas que se deducen de las mismas.

Palabras clave: Tartessos, fenicios, cerámica grabada, decoración geométrica, El Carambolo.
\end{abstract}

\section{SOBRE LA CERÁMICA CON DECORACIÓN GRABADA GEOMÉTRICA}

Dentro del repertorio vascular tartésico, la cerámica grabada debe incluirse en el grupo de la vajilla fina, ya que el acabado de la superficie se realiza mediante la técnica del bruñido. Pero si comparamos la profusión de su estudio con otras integrantes de esta alfarería fina, observaremos cómo el conjunto de los escasos trabajos centrados, de alguna manera, en ella adolecen de cierta imprecisión y ligereza a la hora de abordar algunos aspectos fundamentales. Quizá esta situación se deba a que, dada su exigua representación en el registro arqueológico, no ha llamado lo suficiente la atención de

\footnotetext{
* Este trabajo se enmarca dentro del Proyecto de investigación de excelencia código HUM-3482, denominado "La construcción y evolución de las entidades étnicas en Andalucía en la Antigüedad (siglos VII a.C. - II d.C.)", bajo la dirección del Dr. D. Gonzalo Cruz Andreotti.
}

\begin{abstract}
With this paper we simply aim to present new data on Tartessian pottery with engravings. Specifically, the evidence documented in the field during campaigns of $\mathrm{Ca}$ rambolo in 2002-2005. We conclude with some thoughts about this ceramic type on its functionality and symbolic content, as well as ethnic implications that can be extracted from them.
\end{abstract}

Key words: Tartessos, phoenicians, engraving pottery, geometric decoration, El Carambolo.

los investigadores, o quizá a que su presencia ha quedado eclipsada por su hermana pintada: la cerámica tipo Carambolo.

La mencionada imprecisión ataca a la cerámica grabada desde el momento mismo en que hacemos alusión a ella a través de su decoración. Existe cierto relax a la hora de dar a esta vajilla una terminología concreta en este sentido. Por citar algunos ejemplos, se puede comprobar en la bibliografía cómo algunos autores la llaman grabada (Chaves y De la Bandera, 1984:152; Aguayo y otros 1985: 299; Escacena y otros 1998; Torres 2002) y otros incisa (González y otros 1995: 215; De la Bandera y otros 1993: 17; Ruiz Mata y otros 1981: 246; Ruiz Mata 1986:545; Ruiz Mata y Pérez 1989:291). Los dos apelativos anteriores son los que usa la mayoría de autores, aunque algunos investigadores han utilizado también el término esgrafiado (Murillo 1994:250; Ruiz Gil y López Amador 2001:56; Esteve 1950:17; Martín Córdoba 1993-94:7). 
Para empezar por el principio, veamos que dice el diccionario de la Real Academia Española ${ }^{1}$ sobre los términos que aquí se tratan. La primera acepción de "grabar" es: "Señalar con incisión o abrir y labrar en hueco o en relieve sobre una superficie un letrero, una figura o una representación de cualquier objeto". Como se puede apreciar el diccionario no hace referencia alguna a la dureza de la superficie en cuestión, tampoco cuando habla de "incisión", que se describe de la siguiente manera: "Hendidura que se hace en algunos cuerpos con instrumento cortante". Sin embargo, es en el término "esgrafiar" donde encontramos una diferencia ostensible en la ejecución de la técnica, siendo su definición la siguiente: “Trazar dibujos con el grafio en una superficie estofada haciendo saltar en algunos puntos la capa superficial y dejando así al descubierto el color de la siguiente".

Atendiendo ahora la literatura específica sobre técnicas decorativas en cerámica, encontramos que, por ejemplo, A. Llanos y J. Vegas analizan en su obra la incisión y el grabado. El primero de estos tipos decorativos se corresponde con el grupo 2 que describen de la siguiente manera: "Trazos conseguidos por la aplicación corrida de un instrumento apuntado bien sobre la pasta tierna o una vez realizada la cocción de la cerámica". Dentro de este grupo los autores especifican el subgrupo 2.4 que se corresponde con la técnica grabada y que definen como sigue: "[...] cuando el trazo es fino y poco profundo. Normalmente esta técnica se realiza sobre pasta muy seca o incluso una vez cocida" (Llanos y Vegas 1974:286). He aquí dos diferencias claras entre ambas técnicas: el estado de la pasta en el momento de realizar la decoración, usándose el término inciso, generalmente, cuando está aún blanda y grabado una vez esta se ha secado o incluso ya realizada la cocción; $\mathrm{y}$, por otro lado, la profundidad de los trazos que componen los motivos, siendo el grabado de menor profundidad y más fino que la incisión, característica ésta consecuencia directa de la anterior.

J. J. Eiroa coincide con los autores anteriores en la descripción que hace de la incisión en su apartado 5.2: "[...] se realiza desplazando sobre la superficie blanda de la arcilla un objeto duro más o menos afilado, de forma que éste genere una línea cuyo diseño responda al diseño de dicho objeto" (Eiroa y otros 1999:182). Esta obra, sin embargo, aunque no hace mención al grabado sí describe otras técnicas decorativas que han servido a veces para nombrar a la cerámica que aquí se

1. Se ha usado la vigésima segunda edición del Diccionario de la Lengua Española, de la Real Academia Española (2001). estudia, y que pueden servir para realizar una aproximación, por exclusión, a una definición más exacta. Es el caso del grafitado, que, según los autores, consiste en "aplicar grafito a la superficie de la vasija antes del proceso de cocción, bien cubriendo toda la superficie o amplias bandas de ésta, o bien realizando motivos por lo general geométricos, como reticulados, triángulos rellenos, rombos, líneas paralelas, etc.” (Eiroa y otros 1999:189). J. J. Eiroa hace mención también al grupo de las esgrafiadas, término ampliamente usado entre algunos investigadores para referirse a las grabadas. Según los autores esta técnica " [...] consiste en el raspado en seco de la superficie previamente tratada con un capa de pintura o engobe que ya ha sufrido un proceso de cocción" (Eiroa y otros 1999:189).

Incluso algunos autores que han realizado tablas de clasificación de decoraciones sobre cerámica, caen en la contradicción con respecto a algunos de estos términos. Por ejemplo, en el trabajo propuesto por R. Maicas: dentro del grupo de la incisión, descrito como "[...] presión continuada de un instrumento apuntado sobre la arcilla tierna", encontramos el subgrupo de esgrafiadas: "[...] incisión muy fina obtenida por presión sobre la arcilla seca o cocida.” (Maicas 1994:19). En una nota a pie de página la autora aclara que a este último grupo se le llama también "grabadas".

De las obras anteriormente citadas se desprende que inciso, grabado, esgrafiado y grafitado no son, en absoluto, términos que podamos aplicar a una misma técnica decorativa, siendo "grabada" la nomenclatura que encaja mejor con la decoración que aquí se estudia y, por tanto, el más oportuno a la hora de referirse a esta cerámica y no crear confusión con otros tipos, ya que los motivos se realizan siempre posteriormente al bruñido y a la cocción del recipiente, resultando trazos finos. En la prehistoria en general, la tradición historiográfica ha aplicado el término grabado siempre a decoraciones aplicadas sobre superficies duras: hueso, roca, etc.

Muchas veces el dibujo realizado mediante grabado ha sido, además, rellenado de pasta roja para realzar el motivo y dar un mejor acabado a la decoración (Blázquez y otros 1970:16; de la Bandera y otros 1993: 17; Ruiz Gil y otros 1990:19; Ruiz Mata y otros 1981:246). Generalmente los motivos que decoran esta vajilla son puramente geométricos. Entre estas composiciones se pueden reconocer algunos motivos que abundan también en la tipo Carambolo. Algunos de estos recursos comunes son muy sencillos y se dan también en cerámicas de otras cronologías y áreas geográficas, como el caso de las sucesiones de triángulos tramados. 


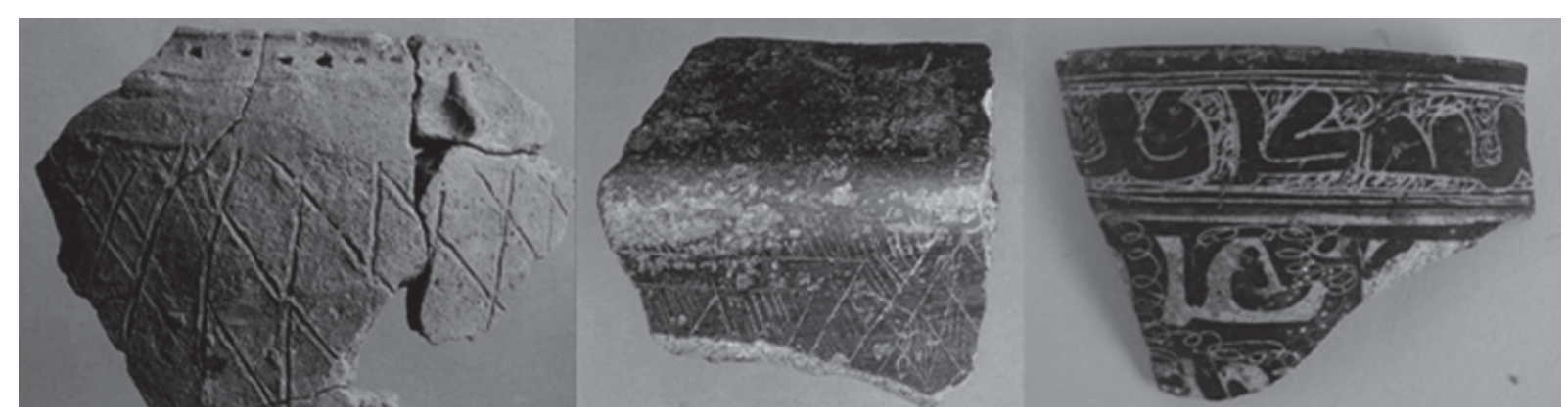

Lámina 1. Diferencias entre las técnicas decorativas citadas. De izquierda a derecha: incisa (fragmento procedente de Acinipo, Ronda, Málaga), grabada (fragmento procedente de Acinipo, Ronda, Málaga) (Martín Ruiz 1995: figs. 229 y 230), esgrafiada (fragmento procedente del antiguo barrio de San Juan de Acre, Sevilla)

Las formas elegidas para plasmar esta decoración suelen ser siempre cazuelas y cuencos carenados de superficies previamente bruñidas, aunque también puede encontrarse en soportes de carrete (Schubart 1979: lám. VII).

En cuanto al área de dispersión, podemos decir que aunque de manera más discreta que otros tipos cerámicos de su familia, se reparte por lo que se ha considerado el territorio tartésico. Empero, también se documenta fuera de los límites clásicos de Tartessos, en colonias fenicias de la costa africana, concretamente en Lixus (Bokbot 1998) y Cartago (Mansel 2011). Se observa una mayor presencia de cerámica grabada en el área de Cádiz. Ante esta circunstancia se ha sugerido que la especie cerámica que aquí tratamos sea característica de dicha zona (González Rodríguez y otros 1995:219); pero, como más de una vez se ha demostrado en la historia de las investigaciones, el lugar donde las cosas aparecen en más cantidad, o lo hacen por primera vez, no tiene por qué ser su zona de origen (Escacena 2000:59 y ss.). Las posibles zonas de producción y vías de redistribución de esta cerámica solo podrán llegar a conocerse con relativa seguridad mediante análisis y comparación de pastas.

En lo que a la cronología se refiere, los fragmentos de cerámica grabada que aparecen estratificados, permiten colocarla dentro del marco cronológico que comparte con el resto de las cerámicas bruñidas tartésicas, tradicionalmente siempre entre los siglos VIII y VI a.C., aunque cabe realizar alguna reflexión para matizar el asunto de la datación de este tipo cerámico. Las recientes aportaciones de referencias cronológicas basadas en análisis de C14 y en la calibración de estas, así como las dataciones asignadas a los niveles fundacionales del propio Carambolo, apuntan a que habría que subir estas fechas, al menos los límites superiores.
Las numerosas concomitancias, en cuanto a formas y decoraciones, entre la cerámica grabada y la pintada tipo Carambolo, así como lo inusual de ambos tipos, pone estas variedades cerámicas en relación casi de manera automática. Sin duda la vajilla que aquí se analiza no debería usarse como servicio de mesa o cocina por el cuidado que se pone en su elaboración y la escasez con la que aparece. El hecho de que en el área de Cádiz se haya documentado cerámica grabada cumpliendo la función de contenedor funerario (Ruiz Mata y Pérez 1989: 291, González Rodríguez y otros 1995:219) proporciona datos acerca de la utilización de este tipo con fines sacros. No hay que olvidar que, al margen del Carambolo, los yacimientos donde se encuentran en un número más elevado de ejemplares, como Acinipo o Doña Blanca no han dado muestras de contener recintos sagrados de tipo templario. Tampoco hay que perder de vista el hecho de que en otras ocasiones se han documentado ánforas y otros tipos de contenedores de ámbito doméstico cumpliendo la función de urna cineraria, sin que este sea el fin concreto para el que habitualmente se fabricaba dicho tipo .

\section{LA CERÁMICA GRABADA EN EL CARAMBOLO}

\subsection{El yacimiento del Carambolo}

Situado sobre el cerro que le da nombre, en localidad la sevillana de Camas (fig. 1), este yacimiento fue excavado en 1958 por J. de M. Carriazo a raíz del hallazgo del famoso tesoro. Las excavaciones en la zona dejaron al descubierto una estructura oval excavada en la tierra virgen, donde aparecieron las piezas de oro, y que proporcionó gran cantidad de materiales. Entre las variedades 


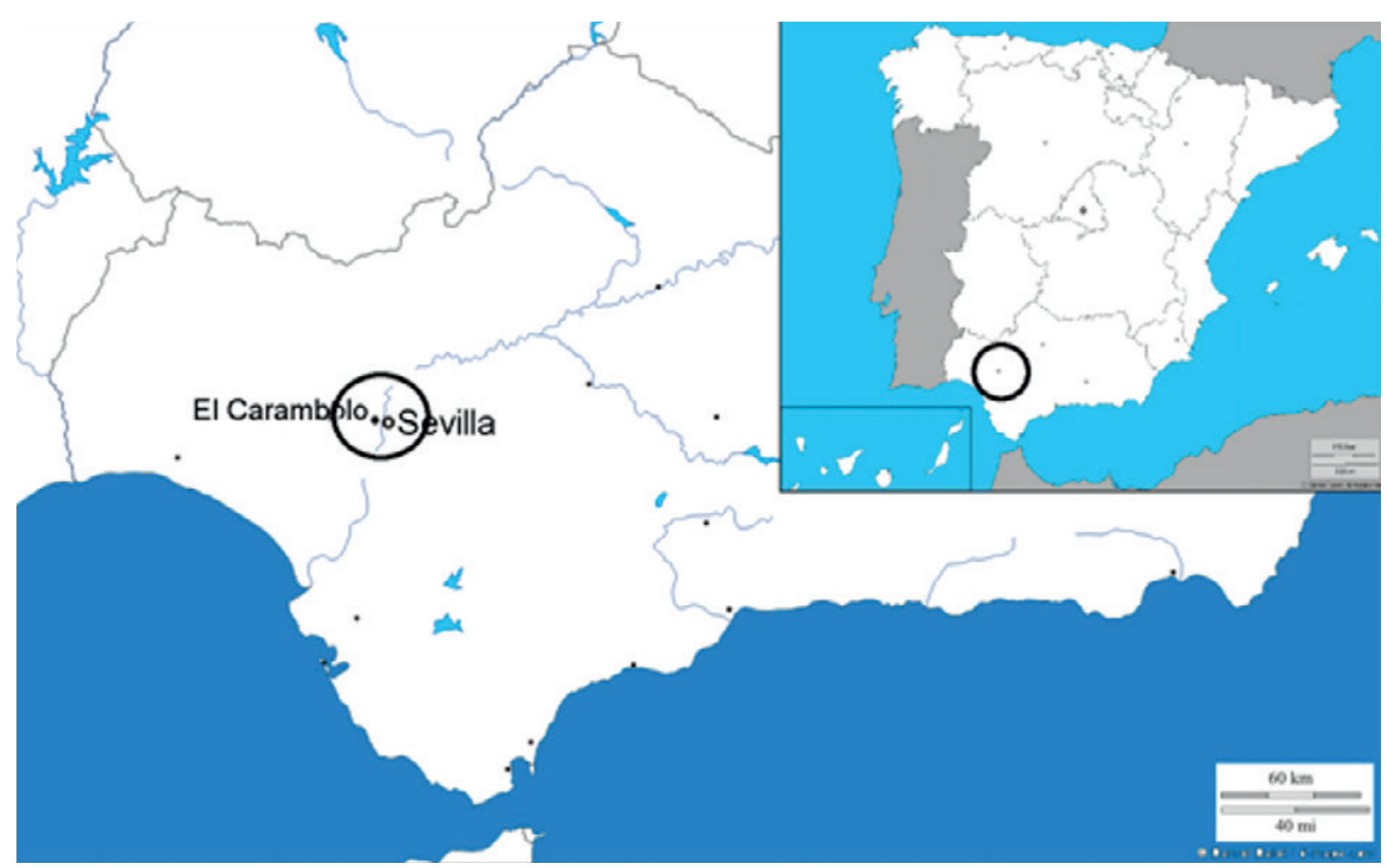

Figura 1. Localización del Carambolo, frente a Sevilla.

cerámicas recogidas en la excavación destacan las conocidas como pintada tipo Carambolo y de retícula bruñida, consideradas posteriormente como fósiles guía de la cultura tartésica. Un poco más abajo, en la ladera noroeste del cerro, se exhumó un conjunto de estructuras de muros rectos que también proporcionó gran cantidad de materiales, entre los cuales se cuenta numerosa cerámica fenicia. Este conjunto fue bautizado como poblado bajo o Carambolo bajo, frente a la estructura oval que se conoció como fondo de cabaña o Carambolo alto.

El yacimiento se interpretó como un poblado tartésico, aunque otros autores posteriormente lo identificaron con un santuario (Blanco 1979; Blázquez 1995). Finalmente, en los últimos años de la década de los 90 del siglo XX, los profesores M. Belén y J. L. Escacena lo reinterpretaron como un santuario fenicio donde se daba culto a Astarté (Belén y Escacena 1998).

Las recientes campañas de excavación en el cerro (2002-2005), con motivo de la construcción de un hotel en la corona del mismo, han aportado relevantes y espectaculares resultados, corroborando algunas de las hipótesis que se habían avanzado en años anteriores. La intervención de urgencia dirigida por A. Fernández Flores y coordinada por A. Rodríguez Azogue actuó sobre una superficie, una vez derribado el edificio de la Real Sociedad de Tiro Pichón, de aproximadamente $5.300 \mathrm{~m}^{2}$ (Fernández Flores y Rodríguez Azogue 2005: 844). Los nuevos datos aportados por estas campañas han sido decisivos, a todos los niveles, para aclarar algunos de los debates más recurrentes en los últimos años sobre el yacimiento, en especial sobre el llamado "fondo de cabaña". La digestión de dichos datos ha puesto sobre el tapete una realidad que hace necesaria la revisión de los paradigmas vigentes durante las últimas décadas sobre el Carambolo en particular y sobre Tartessos en general.

\subsection{Primeros fragmentos}

En la voluminosa y exhaustiva obra de J. de M. Carriazo sobre el Carambolo (Carriazo 1973), el autor no hace alusión clara en el texto a la cerámica grabada. Las únicas referencias que aparecen son las fotografías de la página 543, en las que J. de M. Carriazo se refiere a estos testimonios como "Dos pequeños fragmentos de vasos con decoración que deriva del campaniforme" $\mathrm{y}$ "Pequeños fragmentos de vasos con excepcional decoración grabada con temas geométricos"; y también 

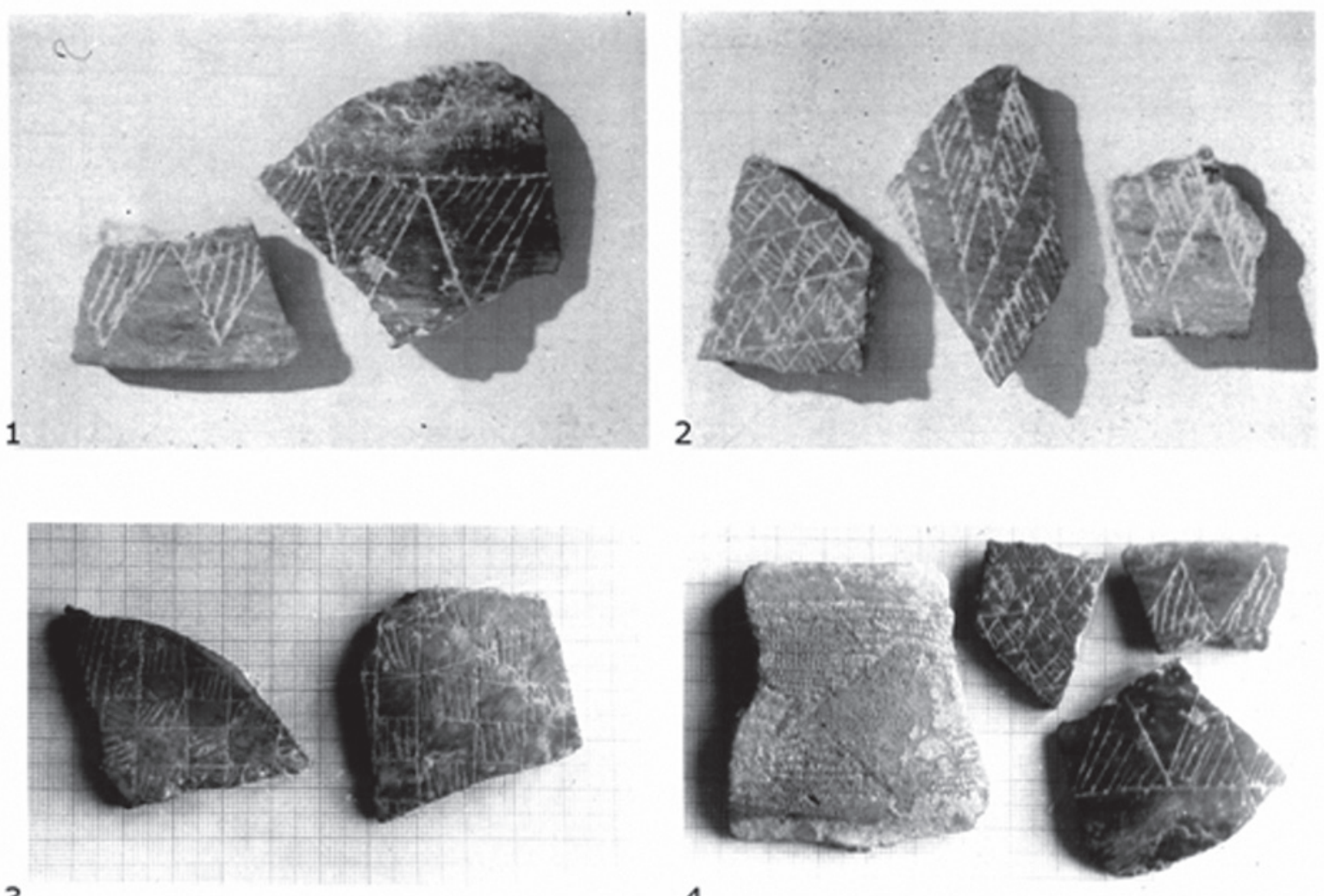

3

4

Lámina 2. Fragmentos de cerámica grabada hallados por J. de M. Carriazo en el transcurso de sus excavaciones.

en la página 565 dice: "Dos fragmentos decorados con ajedrezados con paralelos en Europa central" y "Otros fragmentos únicos de cerámicas de tradición neolítica, el uno con punto y raya" Podemos contar en las fotos ofrecidas por el autor un total de siete fragmentos (lám. 2).

Es difícil averiguar dónde y cómo fueron recogidas las piezas arriba citadas, ya que la única referencia a ellas, como decíamos, son los pies de foto. Tampoco es fácil averiguar si procedían del Carambolo alto o Carambolo bajo ya que las fotos aparecen dentro de los apartados referidos a la cerámica de ambos sectores.

Como se observa en la lámina 2, ninguno de los fragmentos presenta forma reconocible, a excepción - en todo caso- de la pieza situada a la izquierda en la fotografía 1 (que, por otro lado, es la misma que la de la esquina superior derecha de la fotografía 4, pero invertida) que parece un borde, aunque resulta complejo reconocer la forma.

En lo que a la decoración se refiere apreciamos en la lámina la presencia de triángulos tramados, reticulados en ajedrezado y algunos elementos más complejos como triángulos superpuestos tramados con rombos en reserva. Como veremos más adelante, en las piezas documentadas durante las campañas de 2002 - 2005 se registran también estos motivos decorativos.

\subsection{Nuevos testimonios}

Durante las campañas de 2002-2005 se han documentado un total de 35 fragmentos, de los que mostramos una amplia representación en las figuras 2 y 3 . En su gran mayoría se trata de formas que se pueden encuadrar dentro de las llamadas cazuelas carenadas y algunos casos dudosos que probablemente pertenezcan a soportes de carrete o formas de almacenamiento. Las pastas y superficies, bruñidas en su totalidad, muestran una coloración general en varios tonos de castaño. En muchas de las piezas se conserva pintura a la almagra tanto en las líneas de grabado, resaltando así la decoración, como en la superficie interior de los recipientes. 
En cuanto a los motivos y elementos decorativos es muy abundante la sucesión de aspas (fig. 2 CAR-1185$14 ; 2170-17$; 1071-15; 2175-5), de triángulos tramados ("dientes de lobo") simples o con varios niveles superpuestos (fig. 2 CAR-2199-102+106+85; 2547-19; 2096-31; 2544-58; 2199-294; 2125-5; 2094-5), o enfrentados y separados por triángulos mayores con sus lados también tramados (fig. 2 CAR-2199-329; 2199-5; 2199-189). También observamos reticulados oblicuos formando rombos, tramados de manera alterna (fig. 2 CAR-2199-292), o cuadrados tramados de igual modo (fig. 2 CAR-2199-246). Por otro lado, también se dan algunas composiciones más complejas que se comentarán con mayor profundidad en el apartado dedicado a las decoraciones (por ejemplo, fig. 2 CAR-2478-6).

Por otro lado, estos testimonios sí ofrecen información sobre los contextos estratigráficos en los que fueron hallados. La totalidad de las piezas pertenecen al Complejo A, es decir, al área correspondiente al edificio monumental interpretado como santuario (Fernández Flores 2005:77; Fernández Flores y Rodríguez Azogue 2005: 848 y ss.). Concretamente se encuadran dentro de las zonas que los excavadores delimitaron como Ámbitos 2 y 3, principalmente este último, y con algún caso aislado en el Ámbito 1. Dichos espacios se configuran a partir de la fase IV del Carambolo, esto es, tras la primera gran remodelación del santuario (Fernández Flores y Azogue 2007: 109 y ss.). Esta fase se podría situar cronológicamente entre el último cuarto del siglo IX a.C. y principios del siglo VIII a.C. (Fernández Flores y Rodríguez Azogue 2007: 125).

El Ámbito 3, donde se concentra la mayoría de las piezas documentadas, se corresponde con el núcleo central del edificio. La planta es rectangular de aproximadamente $33 \mathrm{~m}$ por $17 \mathrm{~m}$ y se articula de manera simétrica en torno a un patio central abierto, la estancia A-29, que ocupa el espacio en el que se ubicaba la fase anterior (Carambolo V) (Fernández Flores y Rodríguez Azogue 2007:116). En dicha estancia es donde se concentra el mayor porcentaje de cerámica grabada exhumada durante las recientes campañas. Las unidades estratigráficas 2405, 2404, 2509, 2408 y 2549, perteneciente a la fase IV del santuario, son niveles uso y relleno, por deposición continuada de residuos, de la estancia A-29. Similares características de formación presenta la unidad 2199, correspondiente a la fase $\mathrm{II}^{2}$.

2. Agradecemos a Álvaro Fernández Flores y Araceli Rodríguez Azogue, director y coordinadora de las campañas 2002-2005 en El Carambolo, haber puesto a nuestra disposición esta información.

\section{ANÁLISIS DE LOS NUEVOS HALLAZGOS}

\subsection{Formas}

En el aspecto tipológico las piezas documentas, como hemos dicho antes, en su mayor parte se engloban dentro del grupo comúnmente denominado cazuelas carenadas. Se trata de formas abiertas, profundas, con una carena suave (en la mayoría de casos) que divide el cuerpo del recipiente en dos. El tramo superior, de tendencia vertical y con dimensiones próximas a los tres centímetros, da paso a un borde ligeramente exvasado y con labios redondeados. La zona inferior presenta forma hemisférica y solo en uno de los casos (fig. 2 CAR-1071-15) se intuye una base plana; el resto de piezas no la conservan. Los diámetros oscilan entre los 12 y los $20 \mathrm{~cm}$. La pieza CAR-2199-329 (fig. 2) muestra una sección inferior bastante menos profunda y cuya forma se aproxima al casquete de un cuarto de esfera, constituye la excepción en la tendencia.

Formalmente este tipo de cazuelas presenta un perfil óptimo para el desarrollo de la decoración grabada, aprovechando el campo que proporciona el amplio espacio entre la carena y el borde, aunque esto no implica que no se de esta técnica decorativa en formas con un menor desarrollo del borde.

Hay tres piezas que por sus dimensiones y por el desarrollo de sus bordes nos hace pensar que pueda tratarse de grandes soportes de carrete (fig. 2 CAR2096-31, CAR-2199-292, CAR-2478-6). Tipológicamente se vincularían con el tipo D.II de Ruiz Mata (1995:276; fig. 21), fundamentalmente por la tendencia de los bordes, ya que las piezas no conservan un porcentaje de la forma lo suficientemente extenso. La pieza CAR-2405-52 (fig. 3) podría pertenecer al cuerpo de uno de estos grandes soportes de carrete. La tendencia de estos grandes bordes exvasados y la inclinación de los mismos también deja abierta la posibilidad de que pudiera tratarse de recipientes de almacenamiento tipo E.I.b de Ruiz Mata (1995:270; fig.11), parecidos a los vasos à chardon, aunque los diámetros de estas piezas suelen ser superiores a las que aquí presentamos. En cuanto a la relación forma-decoración en esta disyuntiva, se han documentado soportes de carrete con motivos grabados, por ejemplo en la necrópolis de Las Cumbres, en el Puerto de Santa María (Cádiz) y en Morro de Mezquitilla (Málaga). Sin embargo, en lo que concierne a los grandes vasos de almacenamiento, que generalmente no presentan decoración grabada o pintada, solo podemos señalar fragmentos en los que se intuye -al igual que en estos del Carambolo- la 
posibilidad de esta forma, por su diámetro e inclinación de la pared. Ejemplo de esto serían los fragmentos procedentes del Cerro Mariana (Escacena y otros 1998: fig. 2:1; Escacena 2007: 63 y ss.). En Cartago sí se han documentado algunos ejemplares de formas parecidas con decoración grabada, donde al menos un fragmento conserva la zona de inicio del cuerpo de la vasija (Mansel 2011: 72 y s.; fig. 8). Como ya hemos mencionado, es difícil decantarse por una de las dos opciones, dado el estado fraccionario de las piezas en cuestión.

En lo que a las formas se refiere encontramos paralelos a las cazuelas en otros yacimientos del suroeste andaluz y que presentan también decoración grabada. Por ejemplo en la zona de Cádiz vemos formas similares en la necrópolis de Las Cumbres (Puerto de Santa María) (Ruiz Mata y Pérez 1989: 294), o en el material recogido en las prospecciones de Asta Regia (Mesas de Asta, Jerez de la Frontera) (González Rodríguez y otros 1995:219; lám. 2 no 10). Comprobamos que se trata de igualmente de formas profundas, con perfiles relativamente suaves, y con un espacio muy desarrollado entre el borde y la carena, en el que se ubica la decoración.

Ya hemos citado algún ejemplo de soportes de carrete decorados mediante la técnica del grabado, procedentes de la necrópolis de Las Cumbres y de Morro de Mezquitilla (Málaga) (Schubart 1979: lám. VII, c y e; fig. 15 , f y g).

\subsection{Decoración}

La gran mayoría de los elementos decorativos habituales en la cerámica grabada son puramente esquemáticos y geométricos, solo podemos citar dos ejemplos de motivos zoomorfos: un caprino -al cual parece que precede otro- procedente del Castillo de Doña Blanca (Puerto de Santa María, Cádiz) (Ruiz Mata 1988: 46), y un fragmento del Cabezo de San Pedro (Huelva) (Blázquez y otros 1970: lám. XXIX y XXXIII) donde aparece representada una procesión de aves. La aparición de este tipo de animales, principalmente caprinos y aves, es también común en la cerámica tipo Carambolo (Buero 1984; González Rodríguez y otros 1995) y otro tipo de soportes decorados, por ejemplo los huevos de avestruz. La simbología y el mensaje religioso contenidos en estos motivos decorativos, como mencionaremos en el último apartado de este trabajo, es fundamental para ahondar en la funcionalidad de este tipo cerámico.

Un tercer fragmento se podría citar como ejemplo de representación no geométrica ni esquemática mediante la técnica del grabado en cerámica de época tartésica, pero está realizado sobre cerámica gris a torno $\mathrm{y}$, al no pertenecer estrictamente al tipo de vajilla que aquí estudiamos, lo incluimos simplemente como ejemplo de la técnica decorativa del grabado sobre otras series cerámicas. Procedente también del Cabezo de San Pedro (Huelva) (Blázquez y otros 1979:172), el animal representado en este fragmento de cuenco pertenece, en esta ocasión, al mundo mitológico, se trata de un grifo en actitud rampante. El estilo es más naturalista que en los ejemplos citados arriba.

$\mathrm{Al}$ margen de estos casos, los esquemas compositivos de la cerámica grabada, al igual que la tipo Carambolo, se basan en elementos geométricos simples (aspas, triángulos, líneas, cuadros, etc.) cuya repetición y combinación dan forma a motivos más o menos complejos.

En los testimonios exhumados en El Carambolo, durante las campañas 2002 y 2005 , se da una representación relativamente amplia de estos motivos decorativos, como se puede ver en los ejemplos de las figuras 2 y 3 . El más recurrente, sin duda, es el triángulo, presentado de diversas formas (fig. 2 CAR-2199$102+106+85 ; 2547-19 ; 2096-31 ; 2544-58 ; 2199-294$; 2125-5; 2094-5; fig. 4:1), seguido del aspa (fig. 2 CAR1185-14; 2170-17; 1071-15; 2175-5; fig. 4:2). Generalmente aparecen con el interior tramado con líneas oblicuas. En el caso de las aspas es frecuente dejar en reserva el rombo formado por el cruce de las dos franjas que componen el aspa. Ambos elementos decorativos se muestran en motivos más complejos, bien en repetición o bien como parte de una composición más compleja. El uso de estos elementos es muy habitual en la cerámica grabada y en la tipo Carambolo.

También se han documentado, entre las piezas que aquí presentamos, ejemplos de reticulados, tanto horizontales y verticales formados ajedrezados mediante el tramado de cuadrados (fig. 2 CAR-2199-246), como diagonales (con los rombos resultantes tramados de manera alterna) (fig. 2 CAR-2199-292) (fig. 4: 3 y 4).

Se dan también composiciones decorativas más complejas que combinan los elementos simples. Un ejemplo, que encontramos en varias piezas del Carambolo, es el que muestra una sucesión de medias aspas (dos bandas tramadas que se cruzan en un punto, formando triángulos superpuestos, cuyo punto de unión está en reserva), a las que se añade, en los espacios libres entre estas, una sucesión de triángulos tramados y enfrentados (fig. 4:5).

Muy parecido al motivo arriba descrito, encontramos otro algo más complejo. Los triángulos tramados aquí son sustituidos por triángulos superpuestos formados por dos bandas tramadas, dejando igualmente en reserva el 


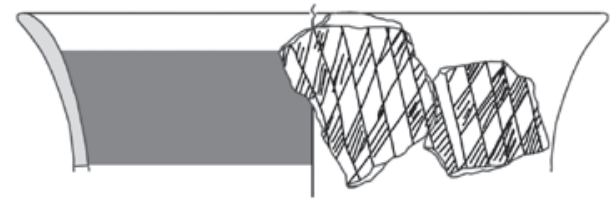

CAR-2199-292

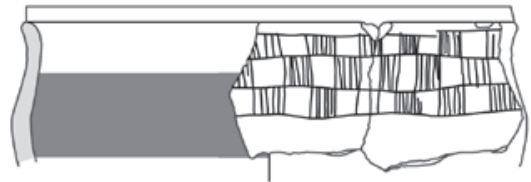

CAR - $2199-246$

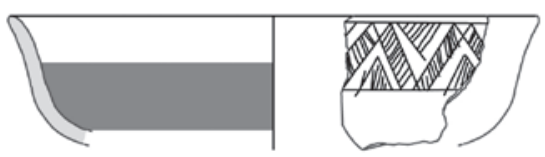

CAR - $2199-329$
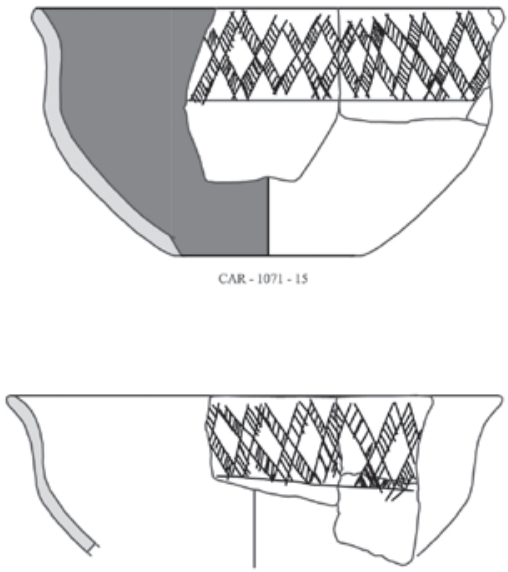

CAR-1185-14
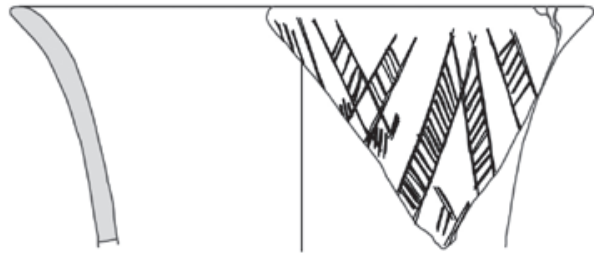

CAR $2487 \cdot 6$

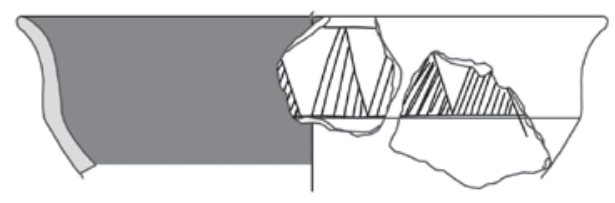

CAR $-2199 \cdot 102+106+85$
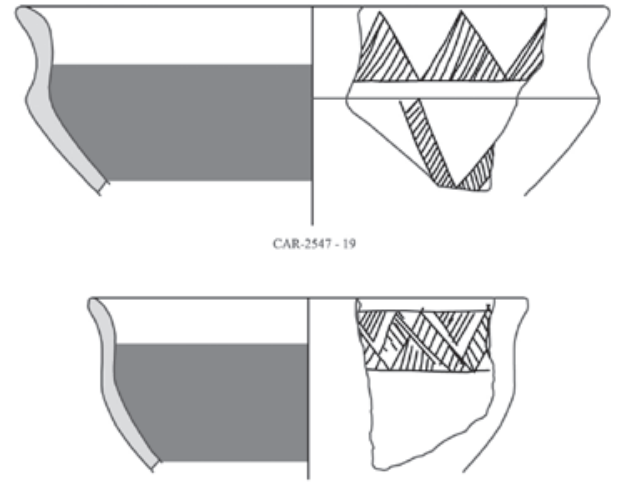

CAR-2199.

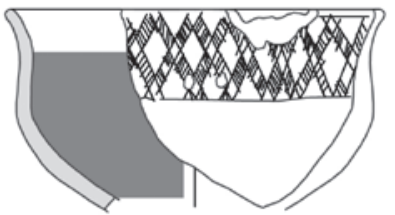

CAR-2170-17

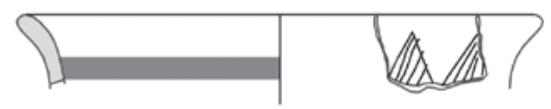

CAR-2544.58

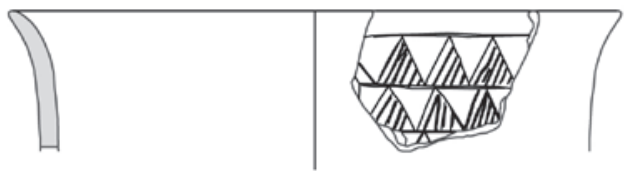

CAR - 2006-3

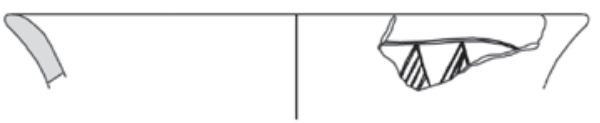

CAR - 2478.5

Figura 2. Nuevos testimonios de cerámica grabada documentados en las campañas 2002-2005 en el yacimiento del Carambolo. 


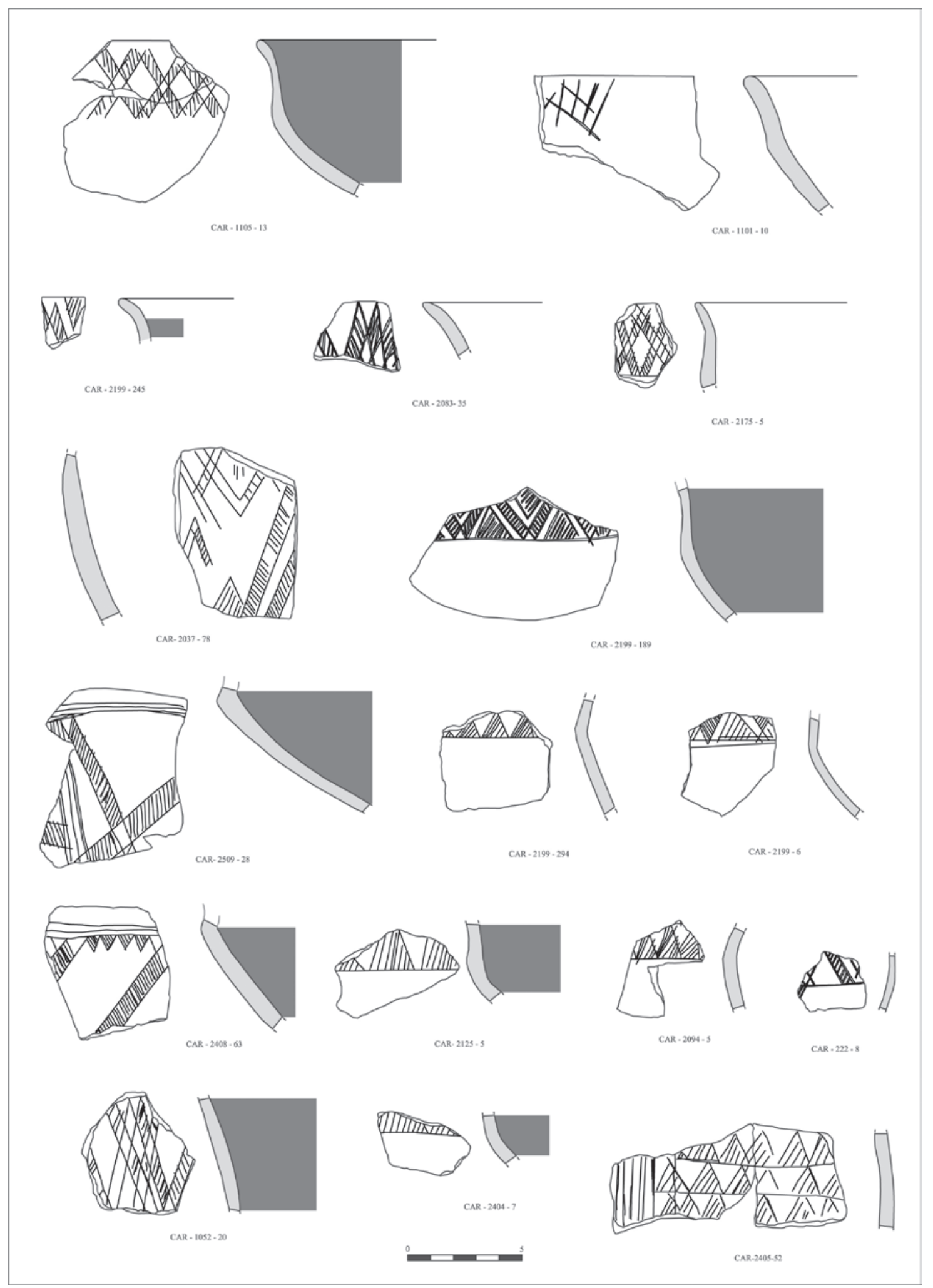

Figura 3. Más ejemplos de nuevos fragmentos de cerámica grabada hallados en las campañas 2002-2005 en el yacimiento del Carambolo. 


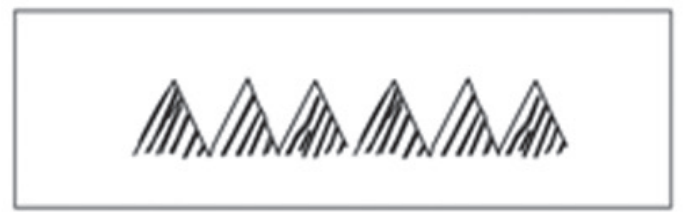

1.- Sucesión de triángulos tramados

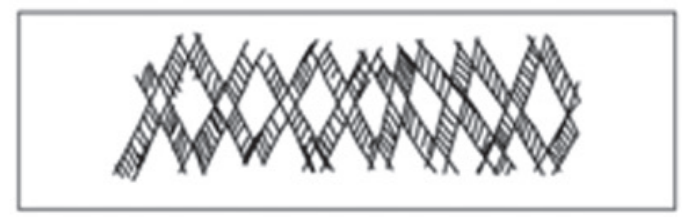

2.- Sucesión de aspas tramadas

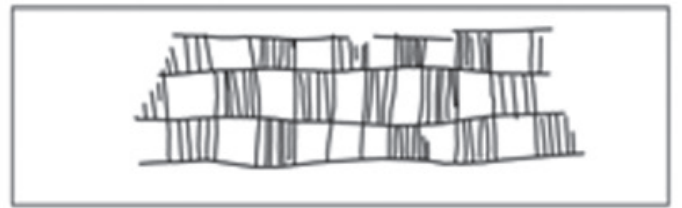

3.- Ajedrezado con cuadros tramados en vertical

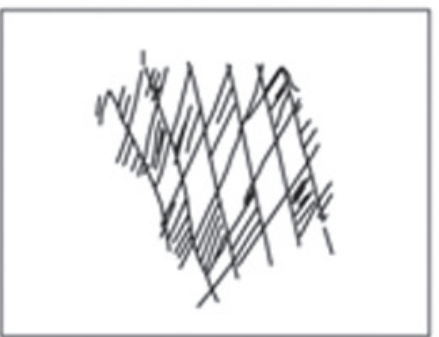

4.- Reticulado oblicuo con rombos tramados

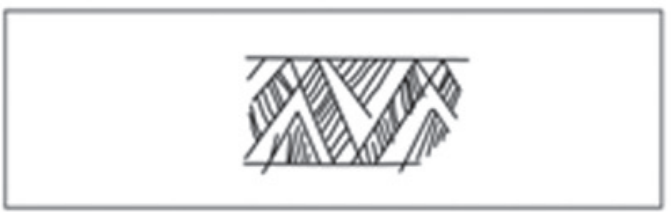

5.- Sucesión de triángulos enfrentados tramados

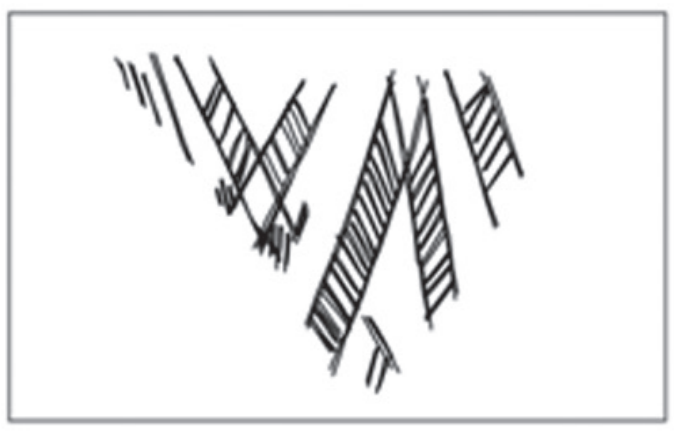

6.- Sucesión de triángulos complejos enfrentados

Figura 4. Selección de motivos decorativos (simples y complejos) documentados en la cerámica grabada durante las campañas 2002-2005.

rombo resultante de la unión de ambas y a los que se adosan unos "flecos" formados por varias líneas. Estos flecos podemos encontrarlos en un testimonio procedente de Estepa (Sevilla) (Blázquez y otros 1970), en la que al triángulo tramado se le adosan también una especie de flecos, también a base de líneas que parten del vértice superior del triángulo. Estos pequeños flecos crean un efecto parecido a unos roleos que, en ocasiones, aparecen en tipo el Carambolo y huevos de avestruz, rematando triángulos.

\section{REFLEXIONES FINALES: ETNICIDAD, FUNCIONALIDAD Y SIMBOLOGÍA}

Hemos tratado en trabajos anteriores las circunstancias ideológicas e historiográficas que propiciaron, basándose en unos fundamentos bastante apriorísticos y precipitados, que el bagaje cerámico documentado en la fosa excavada por J. de M. Carriazo se tomase como la representación material indudable de la cultura 


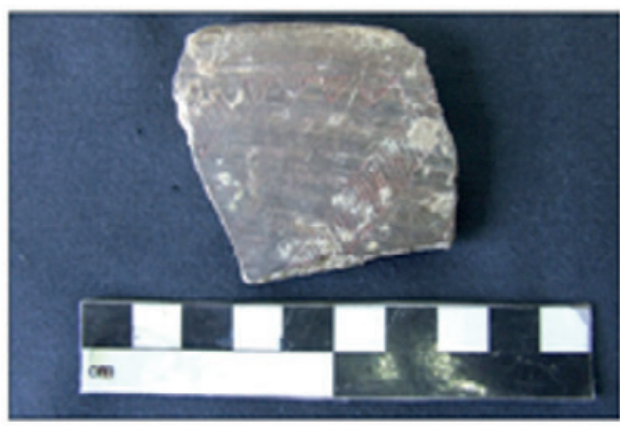

CAR-2408-63

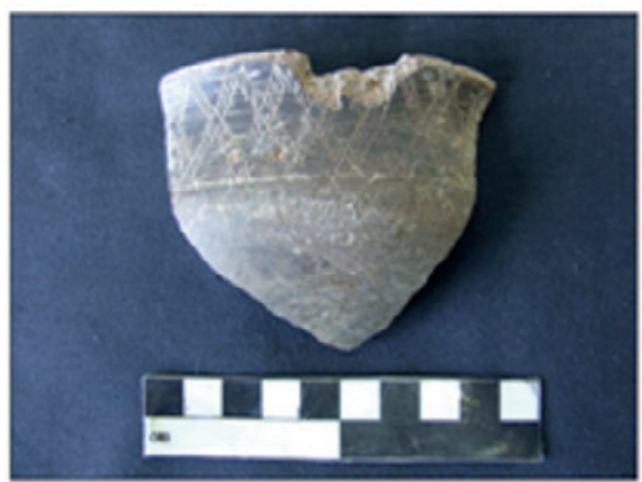

CAR-2170-17

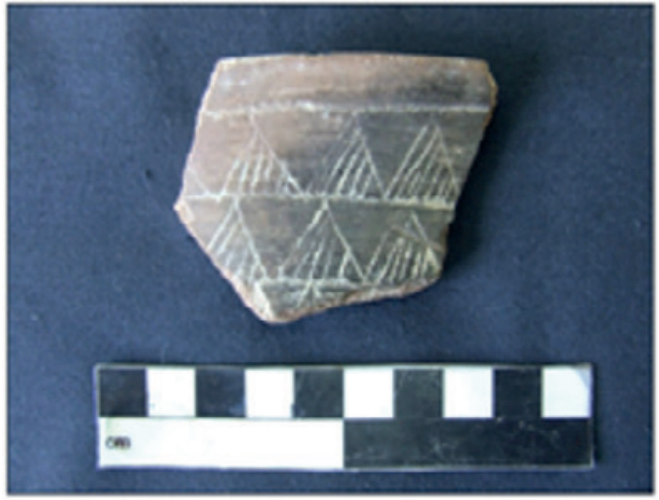

CAR-2096-31

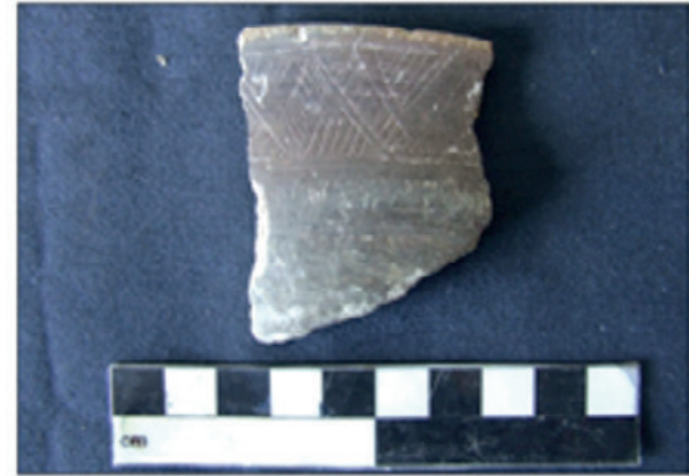

CAR-2199-5

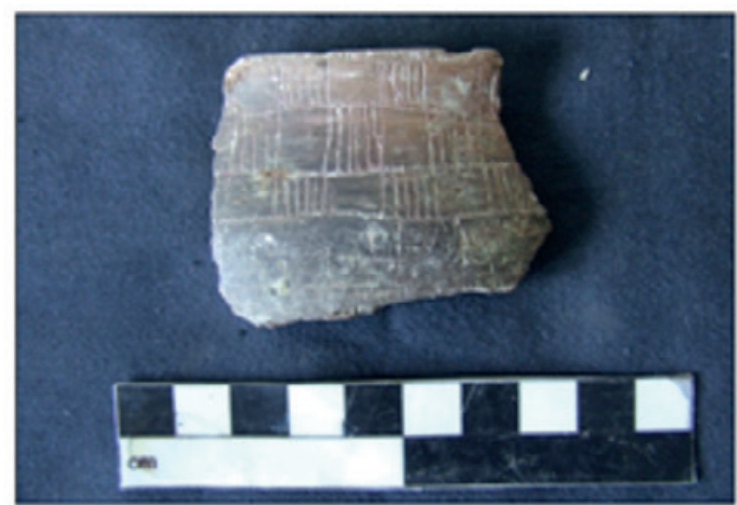

CAR-2199-246

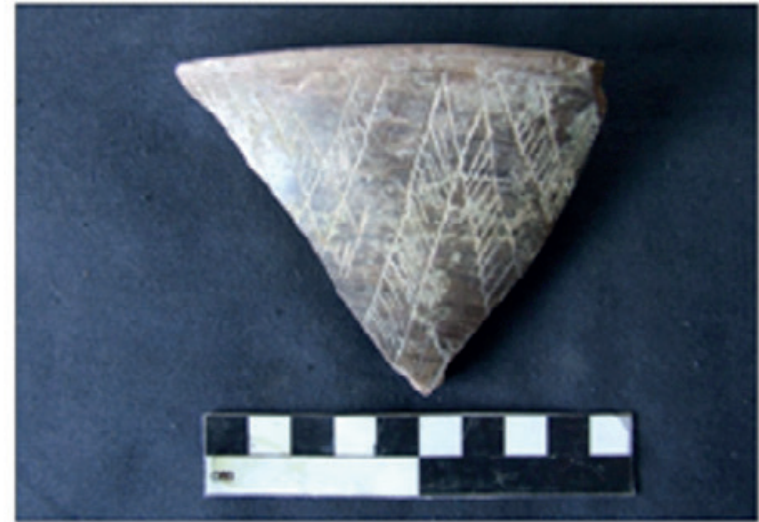

CAR-2468-6

Lámina 3. Ejemplos de la cerámica grabada hallada en las campañas 2002-2005 en el yacimiento del Carambolo.

tartésica, entendida desde un punto de vista indigenista. Igualmente hemos analizado las circunstancias en las que ha nacido, de las cenizas de viejos axiomas historiográficos, una línea de trabajo en la que la consideración étnica de Tartessos queda vinculada con los pueblos de origen oriental asentados en la península ibérica (Casado 2003; 2004; 2010). En este contexto hemos propuesto, también en los citados trabajos, una nueva línea de interpretación, a nuestro modo de ver mucho más coherente, para la cerámica con decoración geométrica tartésica (tanto la grabada como la tipo Carambolo). Esta línea de trabajo se basa sobre todo en la lectura de la decoración y del contenido simbólico de esta, mayormente la de tipo naturalista, y que hace 
alusión a una serie de atributos de una diosa oriental, Astarté para los fenicios del primer milenio, en su advocación, principalmente, como regidora del ciclo vital y señora de la vida y la muerte. Señalamos, una vez más, la relación que guardan los motivos decorativos de la cerámica geométrica tartésica con las cáscaras de huevos de avestruz, de marcado carácter religioso (Casado 2003; 2010). Al margen de esto, la relación de ambos tipos con el yacimiento del Carambolo (de cuya importancia en el mundo religioso de los fenicios del suroeste andaluz ya no cabe duda), acentúa el carácter sacro de estas vajillas. Hay que tener en cuenta, también, que se ha documentado cerámica grabada en contextos funerarios (en la necrópolis de Las Cumbres en el Puerto de Santa María, Cádiz), incluso desempeñando la función de contenedor cinerario. Este hecho encajaría perfectamente con la vinculación de su decoración al entorno religioso de la diosa Astarté. Por todo esto, se podría relacionar la cerámica grabada con algún ritual, dentro de los oficios religiosos de las comunidades fenicias occidentales. Al margen de su presencia, escasa en cualquier caso, en enterramientos, su finalidad podría ser la de contener alguna sustancia o realizar pequeñas ofrendas, tal vez de carácter doméstico.

\section{BIBLIOGRAFÍA}

AGUAYO, P.; CARRILERO, M.; DE LA TORRE, M del P. y FLORES, C. (1985): “El yacimiento pre y protohistórico de Acinipo (Ronda, Málaga). Campaña de 1985", Anuario Arqueológico de Andalucía / 1985, II Actividades Sistemáticas: 294-304. Sevilla, Consejería de Cultura.

BELÉN, M. y ESCACENA, J. L. (1998): “Testimonios religiosos de la presencia fenicia en Andalucía occidental", en El Mediterráneo en la Antigüedad: Oriente y Occidente (Cunchillos y otros eds.) Sapanu. Publicaciones en Internet II, http://www.labherm.csic.es.

BLANCO FREIJEIRO, A. (1979): Historia de Sevilla. La Ciudad Antigua (de la Prehistoria a los Visigodos). Sevilla, Universidad de Sevilla.

BLÁZQUEZ, J.M. (1995): "El legado fenicio en la formación de la religión ibera", en I Fenici: Ieri, Oggi, Domani. Ricerche, scoperte, progetti (Roma 3-5 marzo 1994): 107-117. Roma, Academia Nazionale dei Licei y Consiglio Nazionale delle Ricerche.

BLÁZQUEZ, J. M.; LUZÓN, J. M.; GÓMEZ, F. y CLAUSS, K. (1970): "Las cerámicas del Cabezo de San Pedro", Huelva Arqueológica I.
BLÁZQUEZ, J. M.; RUIZ MATA, D.; REMESAL, J.; RAMÍREZ SADABA, J. L. y CLAUSS, K. (1979): Excavaciones en el Cabezo de San Pedro (Huelva). Campaña de 1977. EAE 102. Madrid, Ministerio de Cultura.

BOKBOT, Y. (1998): "Une céramique à graffito à Lixus", Bulletin d'archeologie Marrocaine, Tome XVIII: 321-323.

BUERO, M. S. (1984): “Los motivos naturalistas en la cerámica pintada del Bronce Final del Suroeste peninsular", Habis 15: 345-364.

CARRIAZO, J. de M. (1973): Tartessos y El Carambolo. Madrid, Ministerio de Educación y Ciencia.

CASADO, M. J. (2003): "Reflexiones sobre la cerámica tipo Carambolo. ¿Un posible axioma de la arqueología protohistórica del Suroeste andaluz?", Spal 12: 285-298. http://dx.doi.org/10.12795/ spal.2003.i12.11

- (2004): La cerámica con decoración geométrica grabada de época tartésica en Andalucía occidental. Memoria de Licenciatura inédita. Universidad de Sevilla.

- (2010): "Nuevas perspectivas para el estudio de la cerámica a mano con decoración geométrica durante el Hierro I en el ámbito del suroeste andaluz", en F. J. García Fernández y O. Rodríguez Gutiérrez (eds.), Tendencias y Aplicaciones en la Investigación Arqueológica. Encuentros de Jóvenes Investigadores 2006-2007. Universidad de Sevilla: 131-146. Sevilla, Dpto. Prehistoria y Arqueología Universidad de Sevilla / Junta de Andalucía.

CHAVES, F. y DE LA BANDERA, M. L. (1984): "Avance sobre el yacimiento arqueológico de Montemolín (Marchena, Sevilla)", Papers in Iberian Archaeology. BAR International Series 193: 141-186. Oxford.

DE LA BANDERA, M. L.; CHAVES, F.; ORIA, M.; FERRER, E.; GARCÍA VARGAS, E. y MANCEBO, J. (1993): "Montemolín. Evolución del asentamiento durante el Bronce Final y el período orientalizante (Campaña de 1980 y 1981)", Anales de Arqueología Cordobesa 4: 15-48.

EIROA, J. J.; BACHILLER, J. A.; CASTRO, L. y LOMBA, J. (1999): Nociones de tecnología y tipología en prehistoria. Barcelona, Ariel Historia.

ESCACENA, J. L. (2000): La arqueología protohistórica del sur de la Península Ibérica. Historia de un río revuelto. Madrid, Síntesis.

-(2007): "Cerámica protohistórica con decoración grabada procedente del Cerro Mariana", en J. Beltrán Fortes y J. L. Escacena Carrasco (eds.) 
Arqueología en el Bajo Guadalquivir. Prehistoria y Antigüedad de las Cabezas de San Juan: 63-71. Sevilla, Dpto. Prehistoria y Arqueología Universidad de Sevilla / Ayto. de las Cabezas de San Juan.

ESCACENA, J. L.; DEL RÍO, A. y LUNA, M. A. (1998): “Cerámica tartésica con decoración grabada. Nuevos testimonios", Anales de Arqueología Cordobesa 9: 9-23.

ESTEVE, M. (1950): Excavaciones en Asta Regia (Mesas de Asta, Jerez). Campaña 1945-46. (Informes y Memorias de la Comisaría General de Excavaciones Arqueológicas 22). Madrid. Ministerio de Educación Nacional.

FERNÁNDEZ FLORES, A. (2005): Tartessos y el Carambolo: Revisión e interpretación del yacimiento a partir de los datos arqueológicos. Memoria de Licenciatura inédita, Sevilla.

FERNÁNDEZ FLORES, A. y RODRÍGUEZ AZOGUE, A. (2005): "Nuevas excavaciones en el Carambolo Alto, Camas (Sevilla). Resultados preliminares", en S. Celestino y J. Jiménez (ed.), El Período Orientalizante (Anejos de Archivo Español de Arqueología XXXV): 843-862. CSIC, Mérida.

FERNÁNDEZ FLORES, A. y RODRÍGUEZ AZOGUE, A. (2007): Tartessos desvelado. La colonización fenicia del suroeste peninsular y el origen y ocaso de Tartessos. Sevilla, Almuzara.

GONZÁLEZ RODRÍGUEZ, R.; BARRIONUEVO, F. y AGUILAR, L. (1995): "Mesas de Asta, un centro indígena tartésico en los esteros del Guadalquivir", Tartessos, 25 años después 1968-1993 (Jerez de la Frontera):215-237. Jerez de la Frontera, Ayuntamiento de Jerez de la Frontera.

LLANOS, A. y VEGAS, J. I. (1974): “Ensayo de un método para el estudio y clasificación tipológica de la cerámica", Estudios de Arqueología Alavesa 6: 265-313.

MAICAS, R. (1994): "Propuesta para el inventario de materiales cerámicos prehistóricos", Boletín del Museo Arqueológico Nacional, tomo XII, $\mathrm{n}^{\mathrm{os}} 1$ y 2: 5-26.

MANSEL, K. (2011): “Cartago y la Península Ibérica en los siglos VIII-VI a.C.», en M. Álvarez Martí-Aguilar (ed.), Fenicios en Tartesos : nuevas perspectivas. BAR International Series 2245 : 69-85. Oxford.
MARTÍN CÓRDOBA, E. (1993-94): “Aportación de la documentación arqueológica del Cerro de $\mathrm{Ca}$ pellanía (Periana. Málaga) a los inicios del primer milenio a. C. en la provincia de Málaga”, Mainake XV-XVI: 5-35.

MARTÍN RUIZ, J. A. (1995): Catálogo documental de los fenicios en Andalucía (Aubet, M. E. Dir.). Sevilla, Consejería de Cultura.

MURILlO, J. F. (1994): La Cultura Tartésica en el Guadalquivir Medio. Ariadna 13-14. Palma del Río, Museo Municipal.

RUIZ GIL, J. A.; PÉREZ FERNÁNDEZ, F.; LÓPEZ AMADOR, J. J. y MONCLOA, A. (1990): "El yacimiento protohistórico de las Beatillas (El Puerto de Santa María)", Revista de Estudios de El Puerto 4: 11-38.

RUIZ GIL, J. A. y LÓPEZ AMADOR, J. J. (2001): Formaciones sociales agropecuarias en la Bahía de Cádiz. 5000 años de adaptación ecológica en la Laguna del Gallo, El Puerto de Santa María. Cádiz, Arqueodesarrollo Gaditano.

RUIZ MATA, D. (1988): “El Castillo de Doña Blanca. Yacimiento Clave de la Protohistoria Peninsular", Revista de Arqueología 85: 34-48.

- (1995): "Las cerámicas del Bronce Final. Un soporte tipológico para delimitar el tiempo y el espacio tartésico", Tartessos, 25 años después 19681993. Jerez de la Frontera, Ayuntamiento de Jerez de la Frontera.

RUIZ MATA, D.; BLÁZQUEZ, J. Mª y MARTÍN DE LA CRUZ, J. C. (1981): "Excavaciones en el Cabezo de San Pedro (Huelva) Campaña de 1978", Huelva Arqueológica V: 149-316.

RUIZ MATA, D. y PÉREZ, C. (1989): “El túmulo 1 de la necrópolis de «Las Cumbres» (Puerto de Santa María, Cádiz)", en Aubet, M. E. (coord.), Tartessos. Arqueología Protohistórica del Bajo Guadalquivir. Sabadell, Ausa.

SCHUBART, H. (1979): "Morro de Mezquitilla, informe preliminar sobre la campaña de excavaciones de 1976", Noticiario Arqueológico Hispánico 6: 175-207.

TORRES, M. (2002): Tartessos. Madrid, Real Academia de la Historia. 